ISSN 1997-5902

\title{
Utilisation des analyses multivariées dans la caractérisation de la sélectivité des filets de capture du mulet jaune (Mugil cephalus, Linneaus, 1758, Mugilidae) dans l'estuaire du fleuve Sénégal.
}

\author{
SARR* Serigne Modou ${ }^{1}, \mathrm{KABRE}$ Jean-André Tinkoudgou ${ }^{1}$ et DIADHIOU Hamet ${ }^{2}$ \\ 1Université Polytechnique de Bobo-Dioulasso, Institut du Développement Rural, Laboratoire de Recherche et de \\ Formation en Pêche et Faune, 01BP 1091BOBO DIOULASSO 01, Burkina Faso. \\ 2 Centre de Recherche Océanographique Dakar-Thiaroye (CRODT), ISRA/HANN BP: 2241, Dakar, Sénégal. \\ *Auteur correspondant ; E-mail : sarmodou@yahoo.fr ; Tél : (00221) 774358017 \\ Original submitted in on $5^{\text {th }}$ June 2013 Published online at www.m.elewa.org on 30th June 2013. \\ https://dx.doi.org/10.4314/iab.v66i0.95006
}

\section{RESUME}

L'objectif de cette étude est de déterminer la sélectivité des filets de pêche utilisés dans l'estuaire du fleuve Sénégal pour la capture de $M$. cephalus en pêche artisanale.

Méthodologie et résultats : un échantillonnage aléatoire utilisant le cree/ survey sur site est fait entre mars 2011 et mars 2013 pendant la saison sèche froide, la saison sèche chaude et la saison pluvieuse dans trois débarcadères (Goxu mbathie, Gueth Ndar et Sor Diagne) de l'estuaire du fleuve Sénégal. Les analyses effectuées avec le logiciel FiSATII ont été étayées par celles multivariées dont l'Analyse des Composantes Principales (ACP) et l'Analyse Factorielle des Composantes (AFC) afin de mettre en évidence la discrimination des populations de poissons capturés et de caractériser la sélectivité des filets. Ceci étant, les résultats à l'examen du plan factoriel des Captures par Unité d'Effort (CPUE) des engins de pêche permettent de distinguer deux grands groupes de filets par rapport à l'axe1. Le premier groupe est constitué de Filets Maillants Dérivant de Surface(FMDS) monofilament en crin, de grandes mailles 56 à $60 \mathrm{~mm}$, de Sennes Tournantes (ST) en nylon multifilaments, de maille $36-40 \mathrm{~mm}$. Le seconde groupe est constitué de FMDS monofilament en crin avec des mailles comprises entre $30 \mathrm{~mm}$ et $50 \mathrm{~mm}$ et de Sennes de Plage (SP) en nylon multifilaments avec des mailles 28 à $40 \mathrm{~mm}$. Finalement les résultats obtenus montrent que d'une part la sélectivité des filets de pêche dépend de la nature du filet, de la maille, de la hauteur de chute, du rapport d'armement, de la saison et de la longueur totale du poisson et que d'autre part les FMDS à grandes mailles (56 à $60 \mathrm{~mm}$ ) sont les plus sélectifs de tous les engins utilisés pour la capture du mulet jaune dans l'estuaire du Fleuve Sénégal. Ils précédent les FMDS à moyennes mailles ( 30 à $50 \mathrm{~mm}$ ) et les ST qui occupent la deuxième position.

Conclusion et application : il est conclu que l'étude de la sélectivité des populations de poissons par les filets à l'aide du logiciel FiSATII peut être couplée avec les méthodes d'analyses multivariées afin de mieux élucider la 
discrimination des populations et de mieux caractériser les différences intrinsèques des taux de captures entre les types de filets de pêche.

Mots clés : Filets, sélectivité, Mugil cephalus, logiciel FISATII, analyses multivariées, fleuve Sénégal.

Use of multivariate analysis to characterize fishing gillnets selectivity of the yellow mullet (Mugil cephalus, Linnaeus, 1758, Mugilidae) in the estuary of the Senegal River ABSTRACT

Objective: The study aims to determine the selectivity of fishing gear used in the estuary of Senegal River to capture $M$. cephalus in artisanal fisheries.

Methodology and results: a random sampling is done between March 2011 and march 2013 during the cold dry season, the hot dry season and the rainy season in three sites of study (Goxu mbathie, Gueth Ndar and Sor Diagne) in the estuary of the Senegal river. A factor analysis is applied to different variables. The results obtained showed that the selectivity of fishing nets depends on the nature of the net, mesh, height of fall, the hanging ratio, the season and of the total length of fish. Interspecific selectivity showed that the gill nets with large meshes ( 56 with $60 \mathrm{~mm}$ ) are the most selective of all fishing nets for $M$. cephalus. They are followed by the medium meshes ( 30 à $50 \mathrm{~mm}$ ) gill nets and purse seines. The small-mesh gill nets (inferior with $30 \mathrm{~mm}$ ), beach seines and cast nets are less selective. The study recommended the use of nets multifilaments biodegradable averages mesh size between 46 and $60 \mathrm{~mm}$ for durable management of stocks of yellow mullets. Conclusion and application: it is concluded from this study that probability of captures methods coupled with multivariates analysis could yield a better discrimination fish assemblage caught and a pinpoint the impact of the characteristics of fishing gears on catch per unit effort (CPUE).

Key words: Fishing gillnets, selectivity, Mugil cephalus, FISATII software, multivariates analysis, Senegal River.

\section{INTRODUCTION}

La pêche au Sénégal est caractérisée par ses spécificités multi-engins et multi-espèces. La capture considérée comme la résultante du choix tactique du pêcheur (choix d'un engin, d'une espèce-cible et d'un lieu) est décrite par l'ensemble du cortège spécifique la caractérisant. Les engins de pêche sont des outils d'exploitation et d'exploration en production halieutique. Ils servent à percevoir la présence du poisson, les conditions de sa capture. Des techniques de plus en plus performantes sont utilisées pour exploiter les ressources halieutiques (Nédélec et Prado, 1990 ; George et al., 1994 ; Gabriel, 2005). L'augmentation de l'effort de pêche par l'utilisation de filets de plus en plus performants et non conformes à la réglementation a favorisé la surpêche, les captures illégales et la destruction des zones de reproduction des mulets jaunes (Gascuel, 1993 ; Sarr, 2010). Quatre grands types d'engins sont utilisés pour l'exploitation des mulets jaunes dans les pêcheries à Saint-Louis au Sénégal. Les filets maillants dérivant de surface (FMDS), les sennes de plage (SP) et les sennes tournantes (ST) sont les principaux, tandis que les filets éperviers (EP) ont une importance moindre pour capturer les mulets jaunes (Dème et al., 2010; Thiao et al., 2012). Pour l'espèce $M$. cephalus, des études ont été menées sur la biologie de l'espèce (Albaret et Legendre, 1985; Okumuş et Başçnar, 1997 ; Lawson et Abayomi, 2010) et sur l'écologie (Vidy et Franc, 1992 ; Ameur et al., 2003 ; Uneke et al., 2010). D'autres travaux réalisés en Mauritanie (Bernardon et Mohamed Vall, 2003) et au Sénégal (Bousso, 1994 ; Le Douguet, 2009) sur les filets ont porté sur la description des engins, les captures en termes de richesse et la production spécifique. Cependant il n'existe pas d'étude qui porte sur la sélectivité des engins de pêche pour les mulets au Sénégal. Notre étude se propose de coupler les analyses de la sélectivité à l'aide du logiciel FISATII avec les analyses multivariées pour la 
caractérisation de la sélectivité des filets de pêche pour M. cephalus dans l'estuaire du fleuve Sénégal en pêche artisanale.

côtière est largement influencée par les remontées d'eaux profondes ou "upwellings" qui proviennent des eaux centrales du sud de l'Océan Atlantique (Cury et Roy, 1991). localisée entre les latitudes $15^{\circ} 45^{\prime}$ et $16^{\circ} 30^{\prime}$ nord et les longitudes $15^{\circ} 40^{\prime}$ et $16^{\circ} 35^{\prime}$ ouest (Figure 1). La zone

\section{MATERIEL ET METHODES}

La zone d'étude : Le fleuve Sénégal parcourt $1700 \mathrm{~km}$ avant de se jeter en mer au sud de la ville de Saint Louis par une embouchure unique. La zone d'étude est

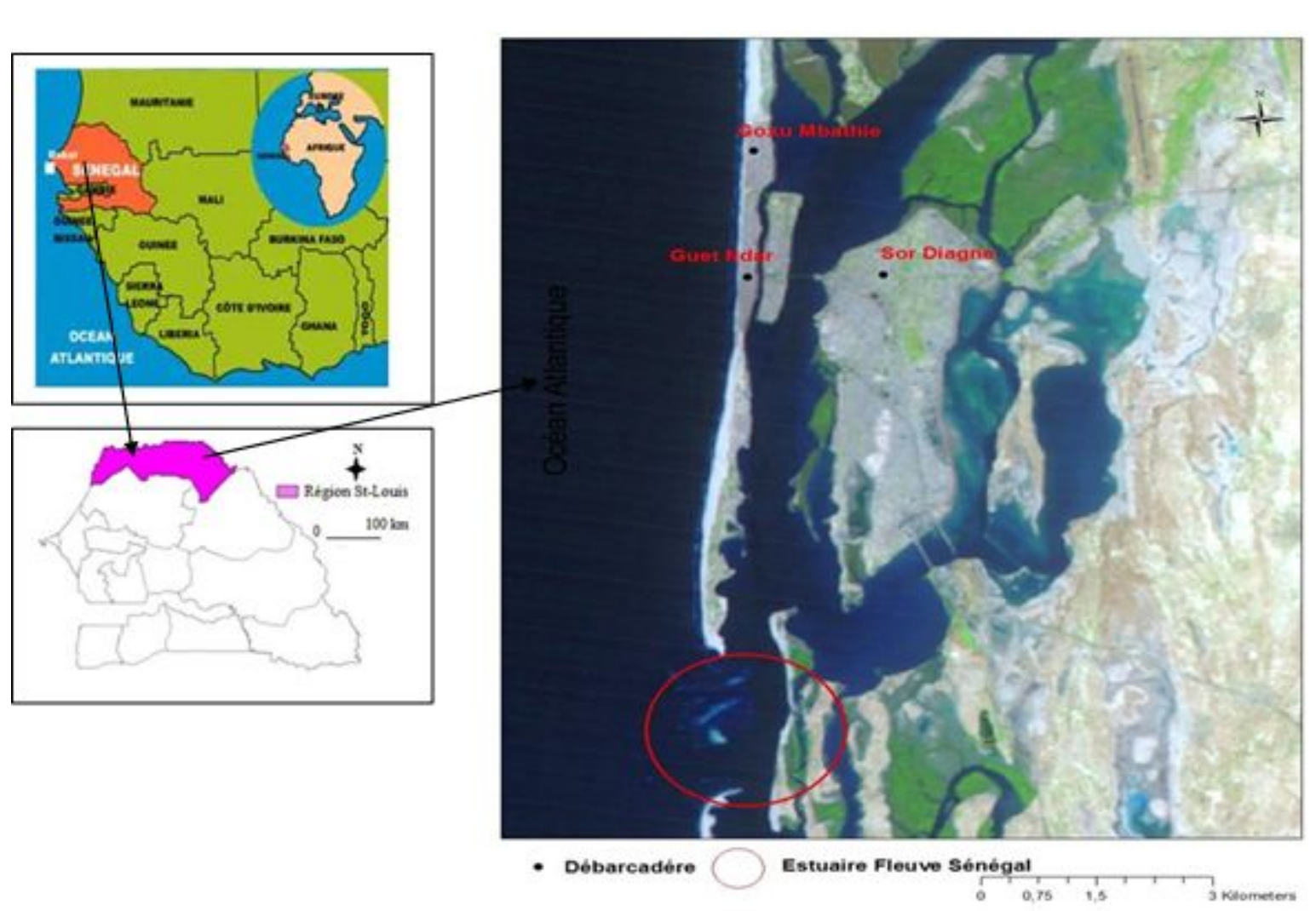

Figure 1 : Carte pour la localisation de la zone d'étude

Collecte des données: Les données de captures, d'effort de pêche et de fréquences de taille sont collectées quotidiennement grâce à un échantillonnage aléatoire entre mars 2011 et mars 2013 pendant la saison sèche froide (SSF), saison sèche chaude (SSC) et saison des pluies (SDP). Le creel interview sur site est utilisé pendant les 96 sorties effectuées sur le terrain au niveau de 21 unités de pêches régulièrement suivies. Cette méthode permet une estimation des captures de pêcheurs, généralement par un programme d'échantillonnage comprenant des entrevues et de l'inspection des captures individuelles, une enquête sur la pêche récréative qui permet de quantifier les débarquements à quai et les quais publics (Bernard et al., 1998). Les données sont collectées à partir des débarcadères de Guet Ndar, Goxu Mbathie pour la pêche maritime artisanale (12 unités de pêche suivies) et de Sor Diagne (9 unités de pêche suivies) pour la pêche artisanale fluviale (figure 1). Les principaux engins de pêche utilisés sont les filets maillants dérivants de surface de maille 30 à $60 \mathrm{~mm}$ en crin monofilament $(\mathrm{C} 1 \mathrm{~F})$ que nous avons codifié (FMDS M<30,C1F; FMDS, M30-50 
C1F ; FMDS, M56-60,C1F) ; des sennes de plage avec des mailles 28 à $46 \mathrm{~mm}$ en nylon multifilament (N4F) codifiés (SP, M28-46, N4F) ; des sennes tournantes en nylon multifilaments de mailles de 36 à $40 \mathrm{~mm}$ codifiés (ST, M36-40, N4F) et des filets éperviers en nylon multifilaments avec des mailles de $36 \mathrm{~mm}$ codifiés (EP, M36, N4F). Le nombre de jours de pêche considéré comme unité d'effort intègre à la fois le temps de route et le temps de pêche selon le type de filets (Gascuel, 1993). Le temps de pêche correspond à la période consacrée exclusivement à la recherche du poisson. II fait apparaître des disparités très remarquables en fonction de l'engin de pêche.

L'effort de pêche est défini ici comme étant le nombre de jours de pêche. Sa formule est :

$E=\frac{j}{j} \sum_{i=0}^{J}\left(e_{i \Gamma}\right)$

$E=$ effort de pêche (nombre de jour de pêche); $\mathrm{J} / \mathrm{j}=$ facteur correctif

$e_{\text {iff }}$ effort de pêche (nombre total de jours de pêche) relevé au cours de la quinzaine dans la zone d'étude $\mathrm{J}=$ nombre total de jours de pêche dans la quinzaine et pour chaque mois $\mathrm{J}=15$ pour la première quinzaine. $P a r$ contre pour la deuxième quinzaine, le nombre de jours de pêche est 15 pour les mois de 30 jours et 16 pour les mois de 31 jours et 13 ou 14jours pour le mois de février ; $\mathrm{j}=$ le nombre total de la quinzaine dans lequel l'effort de pêche a été relevé.

Pour $j=J$, le facteur $J / j=1$ et pour les jours d'indisponibilités (fériées, maladies ou autres contraintes) $j<J$, ce qui justifie ce facteur.

Le lien entre l'opération de pêche (effort de pêche E) et la pression réellement exercée sur les ressources

\section{RESULTATS}

Les efforts de pêche pour $M$. cephalus : Le tableau 1 indique que les pêcheurs qui ont utilisés les FMDS et les ST ont passé plus de temps pour la capture de $M$. cephalus (respectivement $15,3 \mathrm{~h}$ et $14 \mathrm{~h}$ par jour de pêche). Le temps de pêche est de 3,3h par jour de pêche pour les EP. L'effort nominal de pêche pour les mulets halieutiques (mortalité par pêche F) est traditionnellement caractérisé par le coefficient de capturabilité (q):

\section{$F=q \times E(1)$}

En effectuant une simple transformation, on peut également montrer que la capturabilité est également le coefficient reliant la CPUE (Capture Par Unité d'Effort est le ratio entre la capture $C$ et l'effort de pêche $E$ ) et l'abondance du stock considéré $(\mathrm{N})$ :

\section{CPUE= qx $\mathbf{N}$ (2)}

Elle s'exprime en $\mathrm{kg} / \mathrm{J}$.

Analyse des données : Pour déterminer la sélectivité des engins de pêche une étude statistique a été faite en utilisant d'une part le logiciel FiSATII pour la méthode de la probabilité des captures et d'autre part le logiciel $\mathrm{R}$ pour les analyses multivariées. Dans le programme du logiciel FiSATII, les calculs de routines permettent de générer des courbes de sélectivités qui matérialisent les probabilités de capture. Ces résultats ont été par la suite utilisés dans le logiciel $\mathrm{R}$ pour mieux discriminer les populations de poissons capturés et caractériser la sélectivité des filets utilisés. Les variables utilisées ont concerné la longueur des filets, la hauteur de chute, la taille des mailles, la nature des fils (crin ou nylon), le taux d'armement et le mode d'utilisation des filets, les longueurs totales des mulets capturés. Ces variables indépendantes sont utilisées d'abord pour une Analyse des Composantes Principales (ACP), ensuite pour une Analyse Factorielle des Correspondances (AFC) et enfin pour une Classification Ascendante Hiérarchique (CAH) pour déterminer la sélectivité des engins de pêche (Grégoire et al., 1995). Les graphiques ont été réalisés avec les logiciels FiSATII, Statistica7 et R2.11.1 (R Development Core Team, 2008).

jaunes est estimé à 12391 jours de pêche pour les filets utilisés. Les ST et les FMDS totalisent plus de la moitié de l'effort de pêche avec des proportions qui sont respectivement $48,36 \%$ et $28,69 \%$. Le plus faible effort de pêche est noté avec les EP $(3,27 \%)$.

Tableau 1 : Effort de pêche pour différents filets utilisés pour la capture de $M$. cephalus

\begin{tabular}{|l|l|l|l|}
\hline Engin & Temps de pêche (heure) & Effort de pêche (j) & Proportion effort (\%) \\
\hline FMDS & 15,3 & 3556 & 28,69 \\
\hline SP & 9,3 & 2437 & 19,66 \\
\hline
\end{tabular}


Sarr et al J. Appl. Biosci. 2013. Caractérisation de la sélectivité des filets de capture mulet jaune (Mugil cephalus, Linnaeus 1758, Mugilidae) dans l'estuaire du fleuve Sénégal.

\begin{tabular}{|l|l|l|l|}
\hline ST & 14 & 5993 & 48,36 \\
\hline EP & 3,3 & 405 & 3,27 \\
\hline Total & & 12391 & 100 \\
\hline
\end{tabular}

Les Captures par unités d'effort de pêche: La production totale de mulets jaunes débarquée est de 239,39 T pour la période entre mars 2011 et mars 2013. Elle laisse apparaitre des disparités selon le type d'engin et les classes de longueur totale capturées (Tableau 2). La biomasse débarquée par les ST est 96,26T. Les mulets capturés sont principalement des individus mâtures avec des classes de longueurs de 41 à $52 \mathrm{~cm}$ et de 53 à $62 \mathrm{~cm}$ et quelques immatures de 35 à $40 \mathrm{~cm}$. Les filets maillants sont constitués de 3 catégories de mailles qui ont capturé des classes de longueurs différentes. La biomasse débarquée par les FMDS à grandes mailles (56 à $60 \mathrm{~mm}$ ) est $63,45 \mathrm{~T}$ et les individus capturés sont tous mâtures, de classes de longueur 53 à $62 \mathrm{~cm}$ et 63 à $65 \mathrm{~cm}$. La biomasse débarquée par les FMDS à moyennes mailles (50 à $56 \mathrm{~mm}$ ) est de $44,2 T$ et les captures sont constituées en majorité de subadultes et adultes. Les FMDS à petites mailles $(<30 \mathrm{~mm})$ et les EP ont capturé uniquement des individus immatures de longueur totale moyenne de $36 \mathrm{~cm}$. Les ST et les FMDS à grandes et à moyennes sont les plus productifs. Les FMDS à petites mailles, les SP et les EP sont les engins les moins productifs et exercent une forte pression sur les juvéniles.

Tableau 2: Production moyenne (T) par engin selon les différentes classes de longueur totale (en $\mathrm{cm})$

\begin{tabular}{|c|c|c|c|c|c|c|c|}
\hline $\begin{array}{l}\text { Classe de } \\
\text { taille }\end{array}$ & $\begin{array}{l}\text { FMDS, } \\
M<30 \mathrm{~mm}\end{array}$ & $\begin{array}{l}\text { FMDS, } \\
30<M<50\end{array}$ & $\begin{array}{l}\text { FMDS, } \\
56<M<60\end{array}$ & $\begin{array}{l}\text { ST, } \\
\text { M 36-40 }\end{array}$ & $\begin{array}{l}\text { SP, M28- } \\
46\end{array}$ & $\begin{array}{l}\text { EP } \\
\text { M36 }\end{array}$ & Total \\
\hline [17 à $26 \mathrm{~cm}$ & 5,7 & 00 & 00 & 00 & 00 & 1,4 & 7,1 \\
\hline [26 à $34 \mathrm{~cm} \mathrm{[}$ & 9,1 & 00 & 00 & 00 & 00 & 1,2 & 10,3 \\
\hline [35 à $40 \mathrm{~cm}$ & 10,5 & 10,2 & 00 & 31,25 & 3,5 & 0,78 & 56,23 \\
\hline [41 à $52 \mathrm{~cm}$ & 2 & 21,4 & 14,25 & 46,26 & 1,3 & 00 & 85,21 \\
\hline [53 à $62 \mathrm{~cm}$ [ & 00 & 12,6 & 35,50 & 18,75 & 00 & 00 & 66,85 \\
\hline [63 à $65 \mathrm{~cm}$ & 00 & 00 & 13,7 & 00 & 00 & 00 & 13,7 \\
\hline $\begin{array}{l}\text { Total } \\
\text { production }\end{array}$ & 27,3 & 44,2 & 63,45 & 96,26 & 4,8 & 3,38 & 239,39 \\
\hline
\end{tabular}

Le tableau 2 démontre que dans l'estuaire du Fleuve Sénégal, les productions par jour de pêche exprimées en poids ont présenté des différences significatives selon le test de Kruskal-Wallis ( $p=0,0077)$. Les valeurs moyennes des CPUE (Tableau 3) sont statistiquement différentes selon les tailles des individus, le type d'engin de pêche et la maille des filets. L'analyse des CPUE en fonction des structures de tailles confirme que les classes de longueur [41 à $52 \mathrm{~cm}$ [et [53 à $62 \mathrm{~cm}$ [ sont les plus présentes dans les débarquements avec respectivement $5904,84 \mathrm{~kg} / \mathrm{j}$ et $4483,07 \mathrm{~kg} / \mathrm{j}$. Les CPUE les plus importantes sont obtenues avec les ST, les FMDS à grandes mailles et à moyennes mailles.

Tableau 3 : CPUE en poids (Kg) par jour de pêche selon les classes de longueur totale et le type de filet (Kg/j)

\begin{tabular}{|c|c|c|c|c|c|c|c|}
\hline $\begin{array}{l}\text { Classe de } \\
\text { taille }\end{array}$ & $\begin{array}{l}\text { FMDS, } \\
M<30 \mathrm{~mm}\end{array}$ & $\begin{array}{l}\text { FMDS, } \\
30<M<50\end{array}$ & $\begin{array}{l}\text { FMDS, } \\
56<M<60\end{array}$ & $\begin{array}{l}\text { ST, } \\
\text { M 36-40 }\end{array}$ & $\begin{array}{l}\text { SP, M28- } \\
46\end{array}$ & $\begin{array}{l}\text { EP } \\
\text { M36 }\end{array}$ & Total \\
\hline [17 à 26 cm [ & 372,54 & 00 & 00 & 00 & 00 & 424,24 & 796,78 \\
\hline [26 à $34 \mathrm{~cm}$ & 594,77 & 00 & 00 & 00 & 00 & 363,63 & 958,4 \\
\hline [35 à $40 \mathrm{~cm}$ & 686,27 & 666,67 & 00 & 2232,14 & 376,34 & 236,36 & 4197,78 \\
\hline [41 à $52 \mathrm{~cm}$ & 130,71 & 1398,69 & 931,37 & 3304,28 & 139,78 & 00 & 5904,839 \\
\hline [53 à $62 \mathrm{~cm}$ & 00 & 823,53 & 2320,26 & 1339,28 & 00 & 00 & 4483,07 \\
\hline [63 à $65 \mathrm{~cm}$ [ & 00 & 00 & 895,42 & 00 & 00 & 00 & 895,42 \\
\hline
\end{tabular}


Sarr et al J. Appl. Biosci. 2013. Caractérisation de la sélectivité des filets de capture mulet jaune (Mugil cephalus, Linnaeus 1758, Mugilidae) dans l'estuaire du fleuve Sénégal.

\begin{tabular}{|l|l|l|l|l|l|l|l|}
\hline $\begin{array}{l}\text { Total } \\
\text { production }\end{array}$ & 1784,29 & 2888,89 & 4147,05 & 6875,7 & 516,12 & 1024,23 & 17236,289 \\
\hline
\end{tabular}

Les courbes logistiques (Figure 2) sont caractérisées par trois points remarquables $L_{25}$, $L_{50}$ et $L_{75}$ (Lenfant et al. 2011). Les différentes valeurs obtenues sont consignées dans le tableau 4.

$\mathrm{L}_{25}$ : longueur totale à laquelle $25 \%$ des poissons seront vulnérables à l'engin de pêche $\mathrm{L}_{50}$ : longueur totale à laquelle $50 \%$ des poissons seront vulnérables à l'engin de pêche $\mathrm{L}_{75}$ : longueur totale à laquelle $75 \%$ des poissons seront vulnérables à l'engin de pêche Les ST, les FMDS à grandes mailles et à moyennes mailles ont des probabilités de captures plus élevées que les FMDS à petites mailles, les SP et les EP (Figure 2).
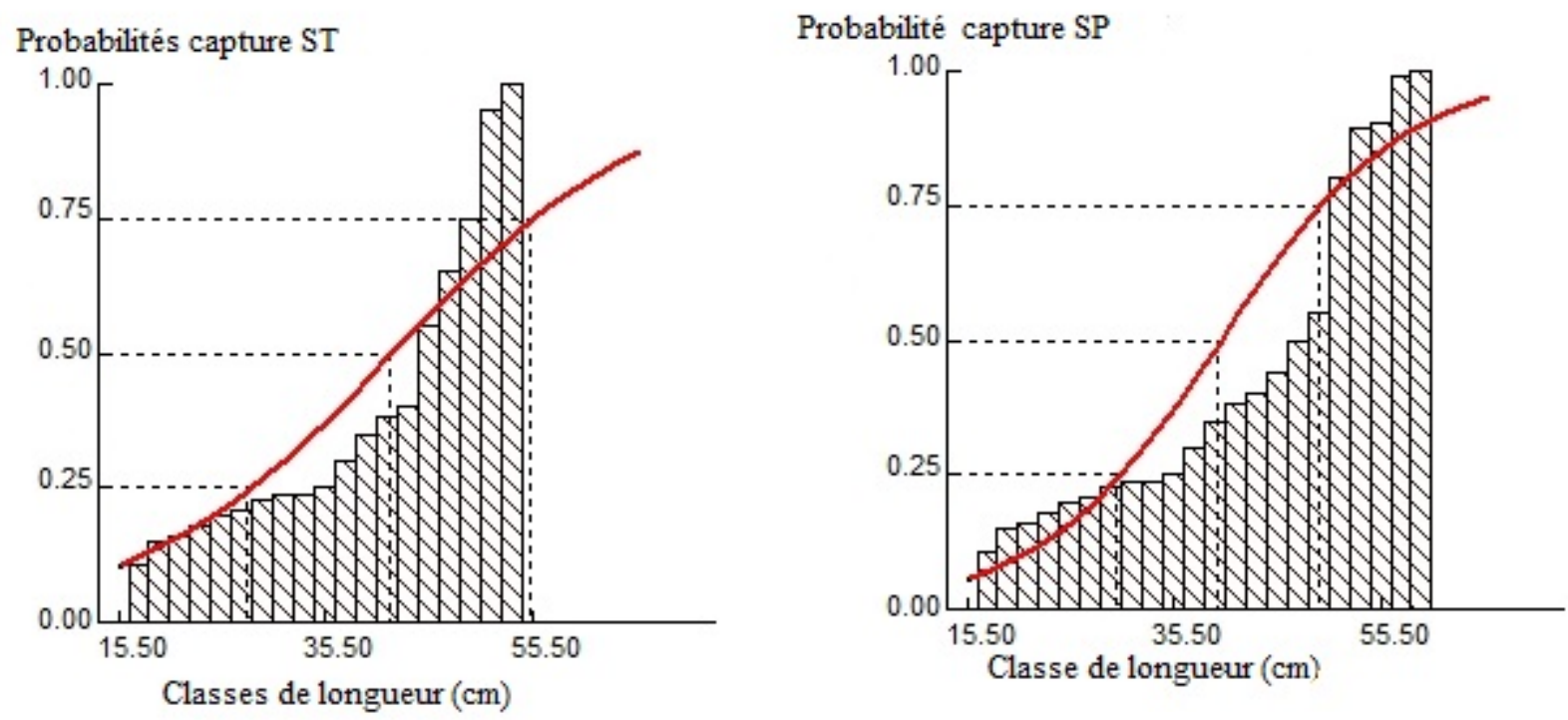

Probabilité capture FMDS
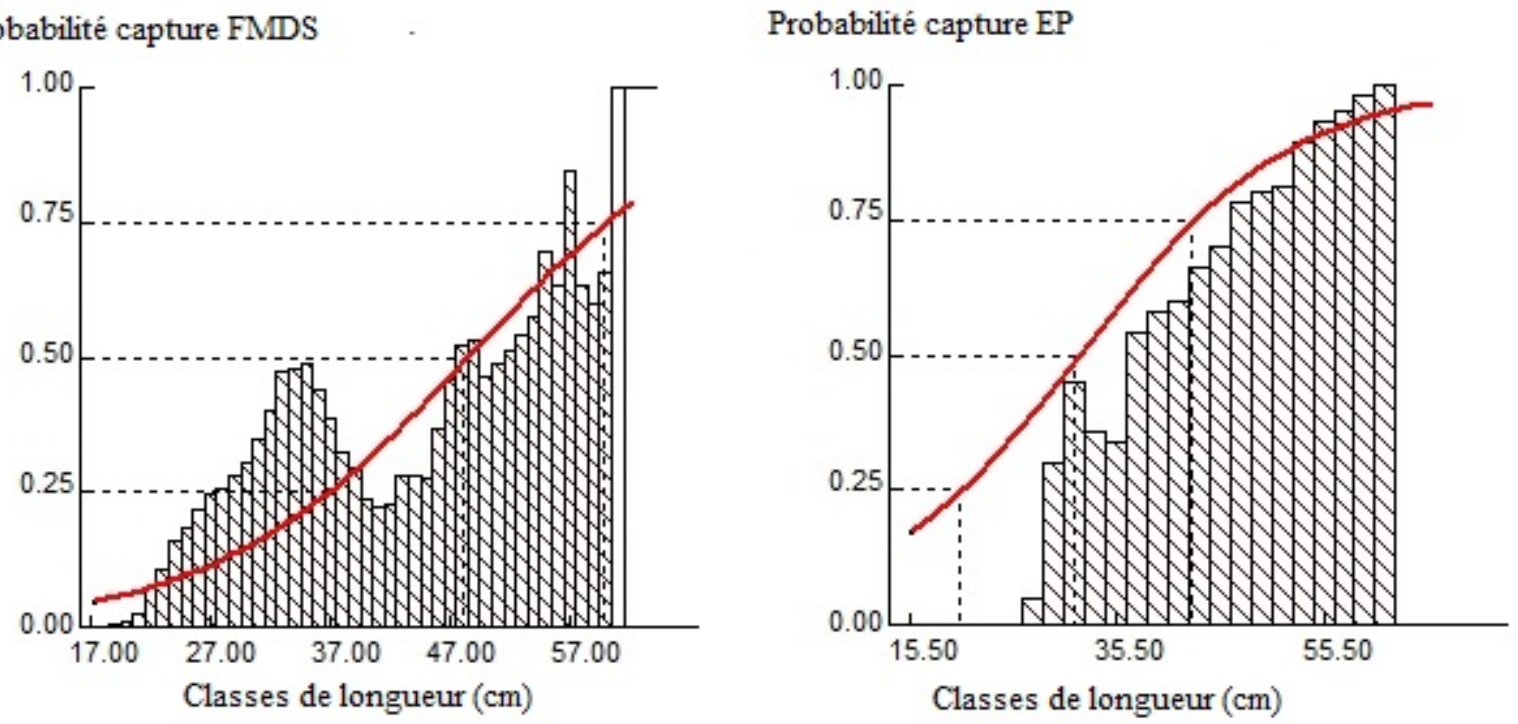

Figure 2 : Probabilités de captures des mulets jaunes dans l'estuaire du Fleuve Sénégal par les sennes (ST et SP) les filets maillants (FMDS) et les éperviers (EP) suivant une courbe logistique.

Selon Sarr et al. (2012), la taille de première maturité sexuelle est de $48 \mathrm{~cm}$ pour les mulets jaunes dans 
l'estuaire du Fleuve Sénégal. La taille $L_{50}$ signifie que $50 \%$ des individus qui ont atteint cette longueur totale et qui entrent en contact avec l'engin de pêche, vont être retenus et les autre $50 \%$ échappent à travers les mailles des filets (Sparre et Vanema, 1996). II peut être illustré par l'exemple suivant : un banc de poissons contenant 18 individus ayant la taille $L_{50}$ du filet EP c'est-à-dire 31,54 $\mathrm{cm}$ entrent en contact avec ce filet il y aura 9 individus qui seront capturés. Dans ce même banc de poissons s'il y a 6 individus qui ont la taille $L_{100}(60 \mathrm{~cm})$ ils seront tous capturés par le filet s'ils entrent dedans. Dans ce même banc s'il y a 20 poissons avec la tailleL 75 (à peu près 40 $\mathrm{cm})$ qui entrent dans le filet il y en aura 15 (75\% de ce groupe) qui seront retenus. Donc dans cet exemple il y a $(9+6+15)=30$ individus qui sont capturés par le filet EP. Pour les FMDS (maille 56 à $60 \mathrm{~mm}$ ), la valeur de longueur totale $L_{50}$ est de $48,18 \mathrm{~cm}$ donc à cette taille $50 \%$ des mulets jaunes qui sont en contact avec ce type de filet sont capturés. Elle correspond aussi à la taille de maturité sexuelle de $M$. cephalus dans l'estuaire du fleuve Sénégal. Ce type de filet est très sélectif et cible principalement les mulets de grosses tailles. Pour les EP, la valeur estimée pour $L_{50}$ correspond à une longueur totale de $31,54 \mathrm{~cm}$ donc $50 \%$ des individus en contact avec le filet à cette taille sont capturés et les autres 50\% échappent à travers les mailles. Les filets éperviers n'ont capturés que des juvéniles. Pour les FMDS à petites mailles $(<30 \mathrm{~mm}$ ) et à moyennes mailles ( 30 à $50 \mathrm{~mm}$ ) et les sennes (ST et SP), les longueurs totales pour lesquelles $50 \%$ des individus qui ont atteint ces tailles et en contact avec les filets sont capturés. Elles correspondent à des subadultes qui n'ont pas atteint leurs tailles de première maturité sexuelle (Tableau 4).

Tableau 4 : La probabilité de capture $L_{25}, L_{50}$ et $L_{75}$ des engins de pêche pour $M$. cephalus.

\begin{tabular}{|l|l|l|l|}
\hline & $\mathbf{L}_{\mathbf{2 5}}(\mathbf{c m})$ & $\mathbf{L}_{\mathbf{5 0}}(\mathbf{c m})$ & $\mathbf{L}_{\mathbf{7 5}}(\mathbf{c m})$ \\
\hline FMDS, M<30mm & 31,91 & 40,49 & 59,84 \\
\hline FMDS, 30<M<50 & 35,35 & 42,91 & 50,48 \\
\hline FMDS, 56<M<60 & 36,51 & 48,18 & 59,84 \\
\hline ST, M 36-40 & 27,92 & 41,65 & 55,39 \\
\hline SP, M28-46 & 29,96 & 39,70 & 49,45 \\
\hline EP, M36mm & 20,28 & 31,54 & 42,80 \\
\hline
\end{tabular}

Efficacité comparée des filets par analyse multivariée: L'analyse multivariée des différents paramètres des filets de pêche a montré l'existence d'autres facteurs qui influencent la sélectivité des filets (Figure 4); tels que la nature des filets qui est caractérisée par les variables, crin à 1 fil $(\mathrm{C} 1 \mathrm{~F})$ et nylon à 4fils (N4F), la maille 28 (M28), la maille (M30), la maille 36 (M36), la maille 40 (M40), la maille(M50), la maille 56 (M56) et la maille 60 (M60). Le modèle validé par le test de sphéricité de Bartlett qui indique une valeur de $x^{2}$ observée $(38,768)$ supérieure à la valeur $X^{2}$ critique $(24,996$ avec $\mathrm{ddl}=15)$ et qui confirme que la corrélation entre les variables indépendantes (mailles des filets, la nature, les dimensions, le rapport d'armement) et la variable dépendante (CPUE), est significative à $95 \%$.
Les deux axes du plan factoriel de l'Analyse des Composantes Principales (ACP) expliquent $76 \%$ de la variabilité totale dont $62 \%$ par l'axe1 et $34 \%$ par l'axe2 (Figure 3). L'examen du plan factoriel des CPUE permet de distinguer deux grands groupes de filets. Le premier groupe est constitué de FMDS monofilament en crin, de grandes mailles 56 à $60 \mathrm{~mm}$, de ST en nylon multifilaments, de maille $36-40 \mathrm{~mm}$. Le seconde groupe est constitué de FMDS monofilament en crin avec des mailles comprises entre $30 \mathrm{~mm}$ et $50 \mathrm{~mm}$ et de SP en nylon multifilament avec des mailles 28 à $40 \mathrm{~mm}$. L'axe2 regroupe uniquement les éperviers qui sont des multifilaments en nylon de maille $36 \mathrm{~mm}$. 


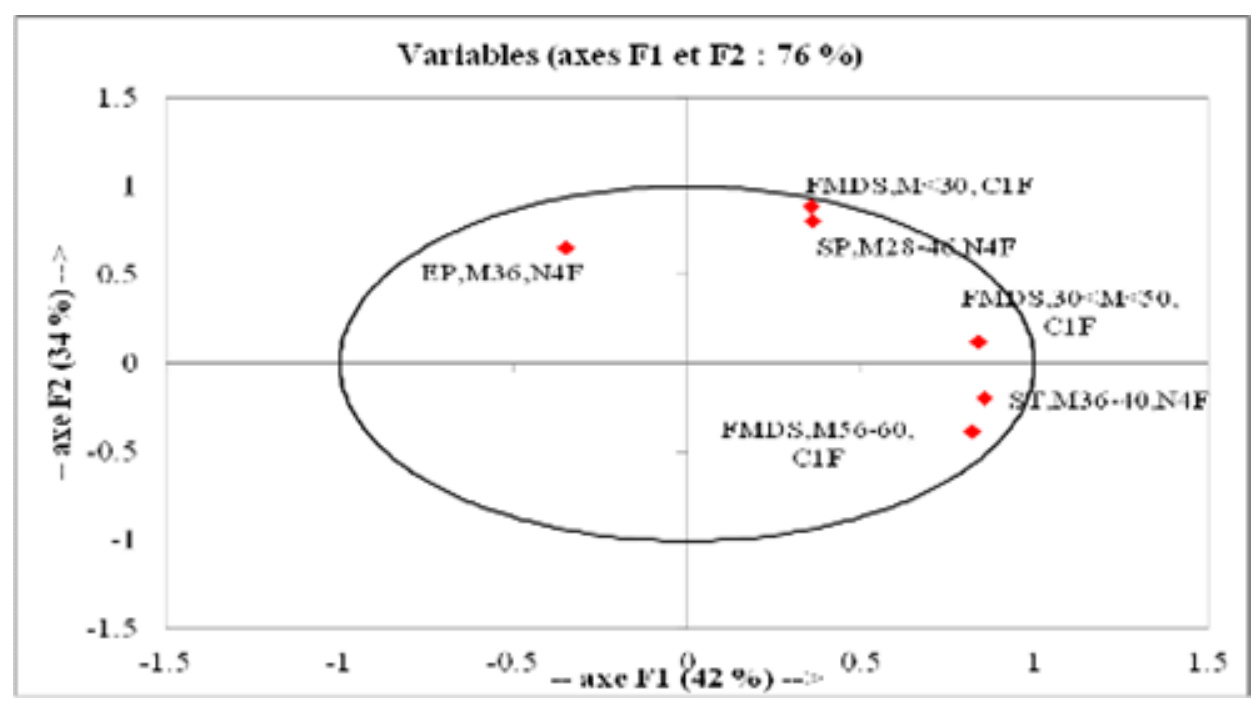

Figure 3 : Analyse des Composantes Principales des CPUE par types de filets de pêche

L'Analyse Factorielle des Correspondances (Figure 4) explique $82 \%$ de l'inertie totale (axe1 $=60 \%$ et axe2 $=22 \%$ ). L'axe1 est un axe de maillage et de sélectivité. II représente à lui seul $60 \%$ de l'inertie totale. Sur cet axe1 les FMDS, M56-60, C1F s'opposent aux FMDS de même nature avec des mailles comprises entre $30 \mathrm{~mm}$ et $50 \mathrm{~mm}$. Les FMDS, M56-60C1F sont utilisés dans la mer pour capturer des mulets de grandes tailles qui ont atteint leurs maturités sexuelles $[53 ; 62 \mathrm{~cm}$ [et $[63 ; 65 \mathrm{~cm}[$; ces filets sont très efficaces pendant les prises en saison sèche froide (SSF). Quant aux filets FMDS, $M<30$, C1F ils sont utilisés dans le fleuve. Les classes de longueurs totales capturées par ces derniers sont situées entre [ $26 ; 34 \mathrm{~cm}$ [ et [35; $40 \mathrm{~cm}$ [ et ils sont efficaces durant la saison sèche chaude (SSC). Les captures sont constituées de juvéniles et de subadultes qui n'ont pas la taille de première maturité sexuelle. Finalement les SP, M28-46, N4F qui sont utilisées dans le fleuve et la partie côtière se sont opposés aux ST, M36-46 N4F utilisées en mer à plus de $10 \mathrm{~m}$ de profondeur. $\mathrm{Ce}$ sont des filets multifilaments confectionnés en nylon avec 4 fils. Les classes de longueurs totales capturées par les SP sont situées entre [26; $34 \mathrm{~cm}$ [et [35; 40cm [. Les mulets jaunes capturés sont des juvéniles immatures. Par contre les ST ont capturé des classes de tailles de poissons situées entre [ $41 ; 52 \mathrm{~cm}$ [. Ils sont constitués principalement de mulets adultes matures et quelques subadultes immatures. L'axe2 de l'AFC oppose un groupe de filets composés de FMDS, M<30, C1F et de SP, M28-46, N4F aux filets EP, M36, N4F. Les SP sont utilisés le plus souvent dans l'estuaire du fleuve Sénégal. Les FMDS de grandes mailles sont efficaces à partir des classes de longueur totale [53 à $64 \mathrm{~cm}$ [ qui ont tous atteint leur maturité sexuelle. Les FMDS à moyenne maille et les ST sont efficaces pour les classes de longueur [41 à $52 \mathrm{~cm}$ [qui regroupent des adultes et des subadultes. Les FMDS de petites mailles et les SP ont capturé des classes de longueur totale [26 à $40 \mathrm{~cm}$ [composées de juvéniles et de subadultes. Les filets EP ont capturé des classes de longueur totale [17à $25 \mathrm{~cm}$ [constitués que de juvéniles qui n'ont pas atteint leurs maturités sexuelles. 


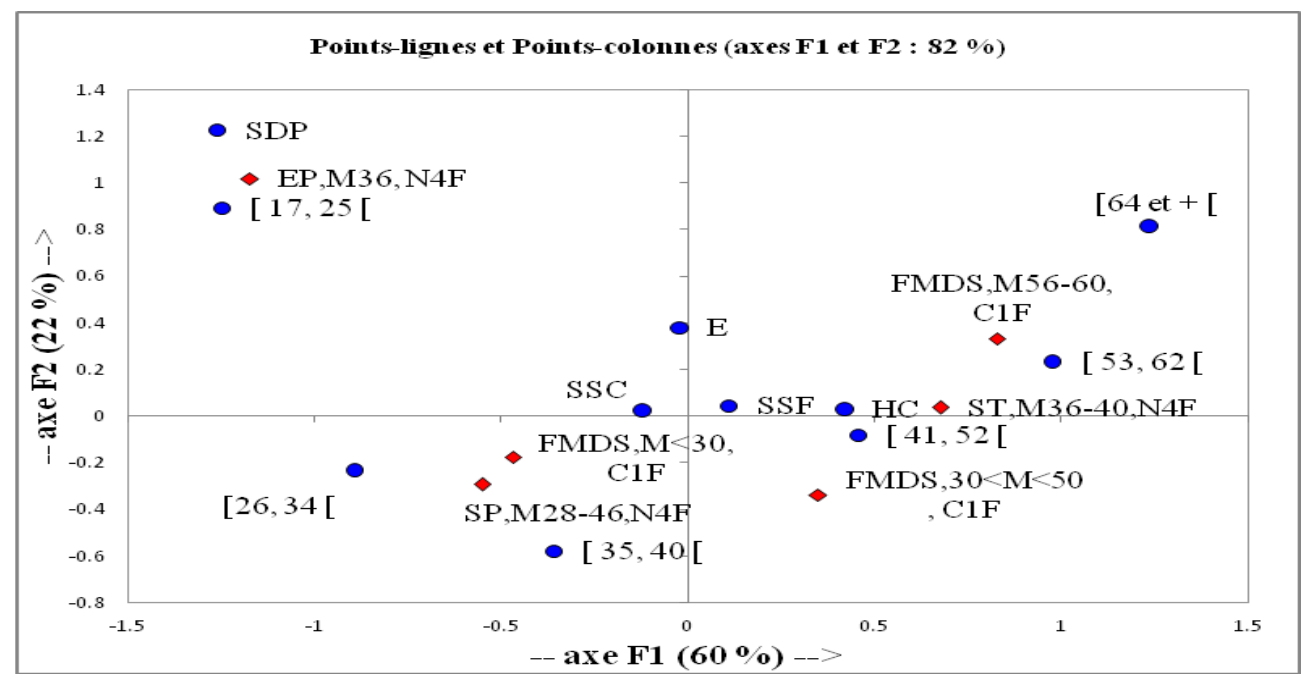

Figure 4 : Analyse factorielle des correspondances par type d'engins de pêche selon la longueur totale des mulets, la saison, la hauteur de chute et le rapport d'armement

Au demeurant, la procédure d'analyse multivariée a permis de représenter les engins de pêche dans un dendrogramme de 6 variables assorti d'une classification ascendante hiérarchique (Figure 5). En effet les données de captures des différents filets de pêches ont permis de faire la classification sur deux axes constitués par la distance d'agrégation (ordonnées) et la distance euclidienne (abscisses) comme distance d'assemblage. Sur la base de similarité, le système a distingué au premier niveau de partition deux groupes (Groupe I et Groupe II). Le groupe I constitué uniquement que de FMDS, M56-60, C1F est conservé et le groupe II s'est subdivisé. Le sous-groupe lla a représenté les FMDS, $30<M<50, C 1 F$ et le sous-groupe llb se subdivise à son tour en deux, Illb1 constitué de SP, M28-46, N4F et Illb21 qui regroupe les ST, M36-40, N4F. Le dernier sousgroupe llb22 est constitué de filets EP, M36, N4F et de FMDS, $\mathrm{M}<30$, C1F.

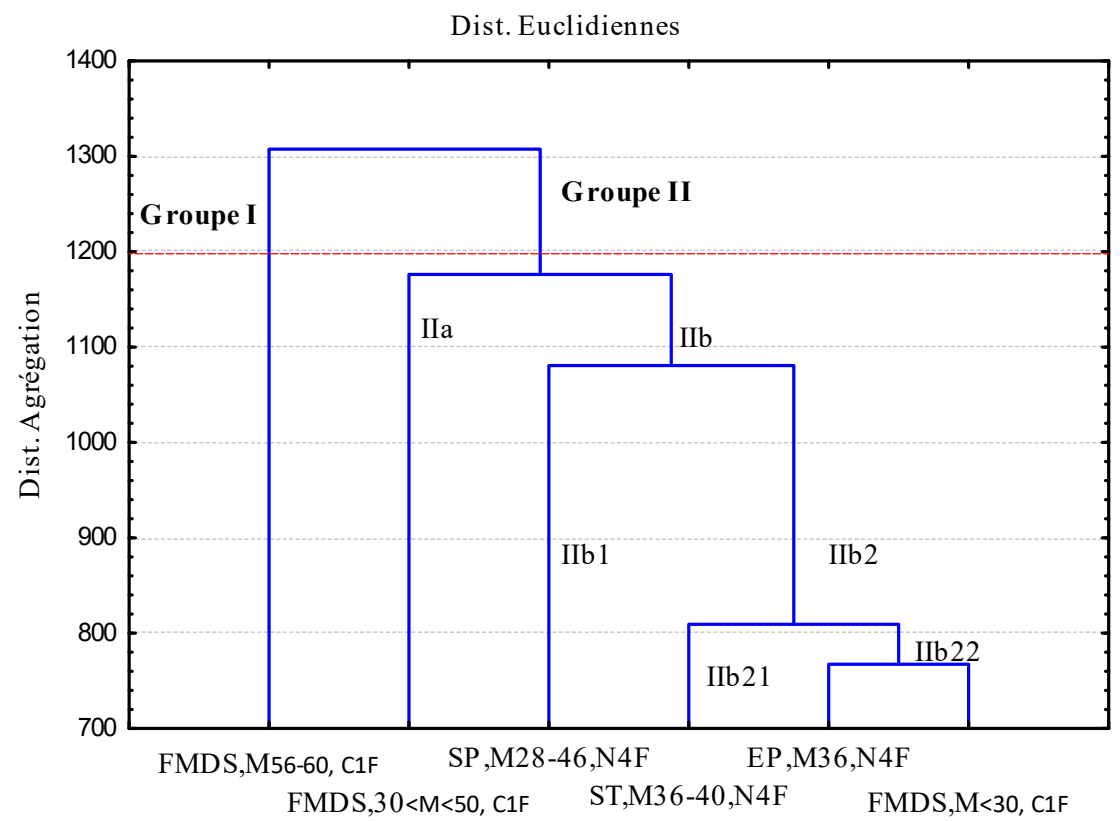

Figure 5 : La Classification Ascendante Hiérarchique (CAH) des engins de pêche ciblant l'espèce M. cephalus dans l'estuaire du Fleuve Sénégal 


\section{DISCUSSIONS}

Les résultats obtenus sur l'effort de pêche de $M$. cephalus (Tableau1) peuvent être comparés aux travaux de Thiao et al., (2012) à Saint-Louis dans la zone économique exclusive. Ces auteurs ont trouvé un effort cumulé de 155101 jours de pêche contre12391 jours pour cette étude. Cette différence significative s'explique par le fait que ces auteurs ont travaillé sur trois espèces de poissons dont le mulet jaune. Pour notre étude, les filets ST représentent $48,36 \%$ de l'effort de pêche et sont suivis par les FMDS $(28,69 \%)$, les SP avec $19,66 \%$ et les filets éperviers $3,27 \%$. Ces différences pourraient s'expliquer par le calendrier d'utilisation des engins qui est influencé par les saisons climatiques distinctes. Les ST et les FMDS sont les engins les plus utilisés et sur une longue période de l'année. Chaque type d'engin de pêche cible une catégorie d'espèces soit des espèces pélagiques ou démersales. En conséquence la longueur totale $L_{50}=48,18 \mathrm{~cm}$ est la taille pour laquelle $50 \%$ des individus sont capturés par les FMDS (maille 56 à $60 \mathrm{~mm}$ ) ; elle correspond à la taille de première maturité sexuelle de $M$. cephalus dans l'estuaire du fleuve Sénégal. Ce type de filet est très sélectif et cible principalement les mulets de grosses tailles. Pour les filets $E P, 50 \%$ des individus qui ont atteint la longueur totale $\left(L_{50}=31,54 \mathrm{~cm}\right)$ sont des juvéniles. Quant aux filets FMDS à petites mailles $(<30 \mathrm{~mm})$ et à moyennes mailles (30 à $50 \mathrm{~mm}$ ) et les sennes (ST et SP), les longueurs totales pour lesquelles $50 \%$ des mulets capturés à ces tailles par les filets sont des subadultes. Ils n'ont pas atteint leur maturité sexuelle. Les filets ST sont utilisés à la fois par la pêche artisanale maritime et fluviale. Ce type de filet est doublement utilisé par les pêcheurs durant toutes les périodes de l'année que sont la saison sèche chaude, la saison sèche froide et la saison des pluies. Selon Ahouansou (2009) les filets ST ne ciblent que les espèces pélagiques qui représentent $81,27 \%$ des débarquements à Saint-Louis. Selon le même auteur, les FMDS sont utilisées dans le fleuve et dans les eaux maritimes côtières pour les espèces pélagiques et bentho-pélagiques. Mais ces filets sont plus actifs pendant la saison sèche froide (Figure 3). Les SP sont utilisés à Saint-Louis, exclusivement pour la pêche artisanale fluviale et côtière. Et elles ciblent des espèces pélagiques surtout pendant la saison sèche chaude. Les éperviers sont les moins déployés et la pêche se fait dans le fleuve à pied ou à pirogue dans la mer. L'influence de l'effort de pêche sur la capture est contrastée si bien que pendant ces deux dernières années, l'effort de pêche a augmenté de $3 \%$. Cependant la CPUE de pêche a chuté de 1,3\%. L'effort de pêche le plus important est observé avec les ST et les FMDS (Tableau 1). Les CPUE les plus élevées sont obtenues avec les ST (6875,7 kg/j) et les FMDS à grandes mailles et à moyennes mailles (respectivement $4147,05 \mathrm{~kg} / \mathrm{j}$ et $2888,89 \mathrm{~kg} / \mathrm{j})$. Les SP $(516,12 \mathrm{~kg} / \mathrm{j})$ et les EP $(1024,23 \mathrm{~kg} / \mathrm{j})$ et les FMDS à petites mailles $(1784,29 \mathrm{~kg} / \mathrm{j})$ sont les moins productifs (Tableau 3) pour la capture des mulets jaunes. Nous avons fait un test de comparaison de moyenne utilisant le test de Friedman; lequel test de Friedman indique que la différence de capture entre les saisons est significative à 95\%. Donc les valeurs moyennes des CPUE selon les saisons sont statistiquement différentes dans l'estuaire du fleuve Sénégal. Cette saisonnalité est à mettre en rapport avec le caractère migrateur périodique des mulets jaunes. A l'approche de sa période de reproduction, une partie de la population de $M$. cephalus quitte le fleuve pour aller en mer (Sarr et al., 2012). Les individus matures sont ciblés par les pêcheurs pour leurs œufs qui sont transformés en poutargue avant d'être exportés dans les marchés européens, américains et asiatiques d'où l'importance des captures à cette période. L'analyse multivariée a montré que la corrélation entre les variables notamment les tailles des mulets jaunes, les mailles des filets, la nature, leurs dimensions et le rapport d'armement est significative.

Les méthodes d'analyses multivariées (ACP, AFC et la classification ascendante hiérarchique) utilisées pour discriminer les populations capturées et caractériser la sélectivité des filets de pêche ont été performantes (figure 3 et figure 4). Notre approche corrobore avec celle utilisée par Grégoire et al., (1995). Les courbes de sélectivité ont montré que les filets de différentes mailles sélectionnent des intervalles de classes de longueurs variables (Figure 2 ; Tableau 4). En outre les filets ont une efficacité considérablement réduite pour les plus petits poissons. Nous pouvons déduire que les plus petits échappent aux filets à cause de leurs petites tailles tandis que les plus grands sont plus exposés aux filets. La distribution des individus capturés par taille montre une nette discrimination des populations selon les saisons et le type de filet; les 6 principales classes de longueur totale $(17-25 \mathrm{~cm} ; 26-34 \mathrm{~cm} ; 36-40 \mathrm{~cm} ; 41-52 \mathrm{~cm} ; 53-$ $62 \mathrm{~cm}$ et $63-65 \mathrm{~cm}$ ) reflètent une distribution particulière en fonction des engins de pêche (Figure 4). La classe de longueur totale $17-25 \mathrm{~cm}$ constituée que de juvéniles domine dans la capture des filets éperviers. Les 
longueurs totales $26-34 \mathrm{~cm}$ et $36-40 \mathrm{~cm}$ sont les plus présentes dans les captures des SP et des FMDS à petites mailles (inférieure à $30 \mathrm{~mm}$ ). Ces trois types de filets ont tendance à capturer des juvéniles dans le Fleuve. Les classes de longueur totale $41-52 \mathrm{~cm}$ sont particulièrement abondantes dans les captures des ST et des FMDS (maille 30 à $50 \mathrm{~mm}$ ). Par contre les classes de longueur totale $53-62 \mathrm{~cm}$ et $63-65 \mathrm{~cm}$ sont plus abondantes dans les captures des FMDS à grande maille (56-60mm). Globalement, les analyses sur la sélectivité montrent que les FMDS à grandes mailles $(56$ et $60 \mathrm{~mm})$ sont les plus sélectifs de tous engins pour l'espèce $M$. cephalus. Ils sont suivi par les FMDS à moyennes mailles (30 et $50 \mathrm{~mm}$ ) et les ST, multifilament en nylon, mailles 36 et $40 \mathrm{~mm}$. Ensuite viennent les SP, multifilament en nylon, mailles 28 et $46 \mathrm{~mm}$. Les filets les moins sélectifs sont constitués de FMDS de petites mailles (mailles inférieures à $30 \mathrm{~mm}$ ) et les EP, multifilaments en nylon, maille $36 \mathrm{~mm}$. Les filets SP, EP et les FMDS à petites mailles sont peu performants et leurs maillages très petits

\section{CONCLUSION}

Les engins de pêche les plus sélectifs sont les filets maillants dérivants de surface à grandes mailles ( 50 à 60 $\mathrm{mm}$ ). Les éperviers et les filets maillants à petites mailles sont les engins les moyens sélectifs pour l'espèce $M$. cephalus. Les sennes tournantes représentent $48,36 \%$ de l'effort de pêche et sont suivis des filets maillants dérivants de surface $(28,69 \%)$, des sennes de plages $(19,66 \%)$ et des filets éperviers $(3,27 \%)$. Les CPUE les plus élevées sont obtenues avec les ST $(6875,7 \mathrm{~kg} / \mathrm{j})$ et les FMDS à grandes mailles et à moyennes mailles (respectivement $4147,05 \mathrm{~kg} / \mathrm{j}$ et $2888,89 \mathrm{~kg} / \mathrm{j})$. Les SP $(516,12 \mathrm{~kg} / \mathrm{j})$ et les $\operatorname{EP}(1024,23 \mathrm{~kg} / \mathrm{j})$ et les FMDS à petites mailles $(1784,29 \mathrm{~kg} / \mathrm{j})$ sont les moins productifs. Les valeurs moyennes des captures par unités d'effort

\section{REMERCIEMENTS}

Les auteurs remerciement vivement le Service de Coopération et d'Action Culturelle de l'Ambassade de la France au Sénégal qui a financé l'étude. Nous remercions aussi M. Mbarack FALL technicien au

\section{REFERENCES BIBLIOGRAPHIQUES}

Anonyme ,1998. Décret $n^{\circ}$ 98-498 fixant les modalités d 'application de la loi portant code de la pêche maritime. Ministère de la Pêche et des Transports Maritimes, République du Sénégal, $27 p$. (quelques fois appelés « moustiquaires ») font que les mulets capturés sont des juvéniles avec des tailles inférieures à $22 \mathrm{~cm}$. L'étude a révélé que ces filets ont exercé une forte pression de la pêche sur les juvéniles et les subadultes. C'est une situation qui met en péril le bon recrutement de l'espèce et favorise ainsi une dégradation du stock de $M$. cephalus dans l'estuaire du fleuve Sénégal. Selon Thiao et al. (2012), les sennes en nylon et les filets maillants monofilament ont contribué à la destruction de l'habitat des mulets jaunes. Et ils sont utilisés sur la frange côtière constituant des zones de frayères des espèces de poissons pélagiques et démersales. Cependant les différents services en charge de la gestion des ressources halieutiques doivent sauvegarder la ressource en appliquant les textes règlementant les captures conformément aux articles 28 et 30 du code de la pêche au Sénégal (Anonyme, 1998). II urge de limiter l'effort de pêche sur le mulet jaune par l'octroie d'une licence qui définisse les quotas annuels.

(CPUE) sont statistiquement différentes selon les tailles des individus, le type d'engin de pêche et la maille des filets et peuvent être facilement discriminés sur le plan euclidien généré par les analyses en composantes principales et les analyses des facteurs par correspondance. Nous pouvons conclure que la méthode des probabilités de capture et l'analyse multivariée sont efficaces pour discriminer les populations des poissons capturés et de caractériser la sélectivité des filets de pêches de M. cephalus dans l'estuaire du fleuve Sénégal. Cette approche d'analyse peut s'appliquer à d'autres espèces pélagiques et démersales de grandes valeurs commerciales.

CRODT pour sa contribution dans la collecte des données et les pêcheurs de la Langue de Barbarie à Saint-Louis pour leur franche collaboration.

Ahouansou MS, 2009. Etude de la Biodiversité et de l'Exploitation des Poissons de la Rivière de Pendjari au Bénin. Thèse en vue de l'obtention du grade de Docteur, Université en Science 
Agronomique de l'Université d'Abomey-Calavi, Bénin, 218p.

Albaret JJ et Legendre M, 1985. Biologie et écologie des Mugilidae en lagune Ebrié (Côte d'Ivoire). Intérêt potentiel pour l'aquaculture lagunaire. Rev. Hydrobiol. trop. , 18(4) : 281-303.

Ameur B, Bayed A et Benazzou T, 2003. Rôle de la communication de la Lagune de Merja Zerga (Gharb, Maroc) avec l'océan Atlantique dans la reproduction d'une population de Mugil cephalus L. (Poisson Mugilidea). Bulletin de l'Institut Scientifique, Rabat, section Sciences de la vie, $25: 77-82$.

Bernard DR., Bingham Allen E. and Alexandersdottir M, 1998. The Mechanics of Onsite Creel Surveys in Alaska. Alaska Department of Fish and Game, Division of Sport Fish, Special Publication $n^{\circ}$ 98$1,136 p$.

Bernardon M et Mohamed Vall MO, 2003. Le Mulet en Mauritanie: Écologie, biologie, pêche et aménagement. Rapport, Programme Régional de Conservation de la zone côtière et Marine en Afrique de l'ouest, $54 \mathrm{p}$.

Bousso T, 1994. Typologie des engins et techniques de pêches artisanales utilisées au Sine-Saloum (Sénégal), Centre de Recherche Océanographique Dakar-Thiaroye, document scientifique numéro 141, 110p.

Cury P et Roy C, 1991. Pêcheries Ouest -Africaine. Variabilité, instabilité et changement climatique. ORSTOM/France 525p.

Dème M, Diadhiou HD, Thiao D et Ndour I., 2012. Bioécologie et exploitation des pêcheries de mulets, courbine et tassergal sur la Grande Côte du Sénégal. Rapport technique, Projet PARTAGE, $38 p$.

Gabriel O, 2005. Fish Catching Methods of the World (4e édition). Blackwell Science, Oxford. $448 \mathrm{p}$.

Gascuel D, 1993. Efforts et puissances de pêche : redéfinition des concepts et exemple d'application. Premier Forum Halieumétrique, Rennes, Session 2 : Relations Efforts, Mortalités, Captures, 23p.

George JP, Deschamps G, 1994. Les engins de pêche passifs. 194p, Ifremer, rapport, DITI/DRV/LORIENT, 194p.

Grégoire F, Huard C, Croteau MN et Lévesque C, 1995. Sélectivité des filets maillants dans la pêche au maquereau bleu (Scomber scombrus L.) I : Baie des Chaleurs. Rapport canadien à l'industrie sur les sciences halieutiques et aquatiques 233, Ministère des Pêches et des Océans, 46p.

Lawson EO et Abayomi AAJ, 2010. Aspects of the biology of grey mullet, Mugil cephalus, in Lagos lagoon, Nigeria. Department of Fisheries, Faculty of Science, Lagos State University, Ojo, Lagos, Nigeria, AACL Bioflux, 3(3): 181-194

Le Douguet L, 2009. Guide de reconnaissance des engins et filets de pêche artisanale utilisés dans les Aires Marines Protégées d'Afrique de l'ouest, rapport, Réseau des Aires Marines Protégées d'Afrique de l'Ouest, 36p.

Nanema P, 1995. Structure de la population et sélectivité du filet maillant de quelques espèces de poissons dans le Lac de barrage de Bangré, mémoire de fin d'étude, Institut du Développement Rural, Université de Ouagadougou, 126p.

Nédélec C et Prado J, 1990. Définition et classification des catégories d'engins de pêche. FAO: Documents techniques sur les pêches, $n^{\circ} 222$. Révision 1. FAO, Rome. 92 p.

Okumuş Î et Başçnar N, 1997. Population structure, growth and reproduction of introduced Pacific mullet, Mugil so-iuy, in the Black Sea. Elsevier Science B.V. Fisheries Research 33:131-137.

R Development Core Team, 2008. R: A Language and Environment for Statistical Computing. $\mathrm{R}$ Foundation for Statistical Computing, Vienna, Austria, ISBN 3-900051-07-0. http://www.Rproject.org

Sarr SM, Kabré AT et Diadhiou H, 2012. Age et croissance de Mugil cephalus (Linneaus, 1758, Mugilidea) dans l'estuaire du fleuve Sénégal. Int. J. Biol. Chem. Sci. 2012, 6(5):2149-2157.

Sarr SM, 2010. Dynamique d'exploitation du Mugil cephalus (Linnaeus, 1758, Mugilidés) dans l'estuaire du Fleuve Sénégal en pêche artisanale. Mémoire de master recherche, Université Polytechnique de Bobo, Burkina Faso, 68p.

Scherrer B, 1984. Biostatistique. 1ère édition. Gaëtan Morin éditeur, Chicoutimi, Canada, 850p.

Sparre P and Venema SC. 1996. Introduction à l'évaluation des stocks de poissons tropicaux. Première partie : Manuel FAO. Document technique sur les pêches numéro 306/1 Rev.1, Rome : 420p. 
Thiao D, Diadhiou H, Dème M, 2012. Evaluation de l'effort de pêche et des captures hors ZEE sénégalaise par la pêche artisanale. Projet USAID/COMFISH, Sénégal, rapport technique à mi-parcours, 50p.

Uneke BI, Nwani CD, Okogwu O et Okoh F, 2010. Growth, mortality, recruitment and yield of Pellonula leonensis Boulenger, 1917, (Osteichtyes : Clupeidea) in Tropical flood River system. Journal of Fisheries International $1(5): 19-26$.
Vendeville P, Rosé J, Viera A et Blanchard F, 2008. Durabilité des activités halieutiques et maintien de la biodiversité marine en Guyane, Rapport final Laboratoire Ressources Halieutiques de Guyane, Ifremer 236p.

Vidy $\mathrm{G}$ et Franc J, 1992. Saisons de présence à la Côte des alevins de muges (Mugilidae) en Tunisie. Hilton Head, South Carolina, U.S.A, Cybium, 16(1): 53-71. 